\title{
Finding a picture's station point: Reconstructing virtual space when the usual way will not work
}

\author{
THOMAS O. HALLORAN \\ University of Maryland University College, Asian Division, Yokota, Japan
}

\begin{abstract}
To reconstruct the space represented in a photograph or other picture made in perspective, its vantage point, or station point, must first be found, and the usual way to find this point requires that the picture show a rectilinear grid or at least one grid element. The station points of the many pictures that lack any such grid may be found by a new method presented here, on two conditions: The line of sight, from the original observer to the scene, must be determinable, and, within the scene, there must be at least one quantitative spatial relation involving both a depth extent and an extent laterally (breadth or height), the 3-D value of which is known. Researchers may use either the graphic (projective) or the computational (algebraic) procedures of this new "sightline" method, in reconstructing the spaces of pictures used as stimuli.
\end{abstract}

When the experimental stimulus is a picture, we often want to know the geometric properties of the spatial layout it represents. For instance, to find out whether we correctly perceive what we see in a perspective display, we need to know what the distances, depths, and directions are in the virtual space seen "behind" the display. And to know these things without measuring the real 3-D scene, we must reconstruct its properties from its depiction.

To reconstruct the virtual space of a picture made in correct perspective (e.g., photographs and much of Renaissance pictorial art), we first must locate the picture's station point, which is the "taking" or "making" viewpoint, where the camera or the artist's eye had been. (For purposes such as positioning observers in front of a stimulus picture, the entire reconstructive goal might be simply to find this original station point.) Until now, we could find this point only for pictures that happened to contain an element or pattern of known spatial proportions extending in depth (Greene, 1983; Sedgwick, 1980) or for certain three-point perspectives ${ }^{1}$ (Greene, 1983). With the sightline approach offered here, we can find the station point in many pictorial situations for which the usual ways will not work. The approach has uses in vision studies and in historical reconstruction, and it may have implications for perceptual theory and machine vision, as briefly discussed at the end of this article.

\section{Alberti's Ground-Plane Grid}

Alberti (1435/1991) presented two methods, now called Alberti's grid and Alberti's veil, for making an ac-

\footnotetext{
The author thanks Ronald E. Mathis for important insights and consultation throughout this project. He also thanks Harold A. Sedgwick, Richard Greene, and an anonymous reviewer for advisory comments that enormously improved the clarity of presentation of these ideas. Correspondence should be addressed to T. O. Halloran, 2605 Range Ave., Apt. 67, Santa Rosa, CA 95403 (e-mail: hallorantom@hotmail.com).
}

curate perspective drawing. His grid method (Alberti, $1435 / 1991$, pp. 56-58) was a way of projectively drafting into the picture a pattern of squares (he suggested paving tiles) laid out on level ground, so that the pattern became depicted in correct perspective. Then, objects and people were painted appropriately in place on the depicted tiles, according to the positions they had on the real pavingtile pattern in space. As more or less of a reversal of this method of making a picture, there evolved the traditional ground-plane-grid method, described in Greene (1983), for rediscovering the gridded picture's station point. After the station point is found, the grid may also be used to reconstruct the picture's virtual space (Steadman, 2001), much as pieces shown in a photograph of a chessboard could be located on the real board.

A ground-plane grid consisting of a single square or rectangle, or sometimes even a circle (Greene, 1983), is sufficient for finding the station point, and the grid need not be horizontal. Figure 1 shows the picture of a cubeshaped box with the open side facing the viewer, and it also shows the plan and elevation views of the box, from which this picture was drafted by projection, as indicated. Considering the floor (and/or the ceiling) of the box as a square grid of one element, Figure 2 shows the construction on the picture of distance points, which are points where diagonals, drawn across the grid and then extended beyond it, cross the horizon. (The vertical sides of the picture of the box might instead have been used to construct such diagonals and distance points, though this was not done.) In a one-point or two-point perspective, the distance points will specify where the line of sight (LOS), which is a perpendicular from the station point, intersects the picture plane, and they also specify the distance, $d$, along the LOS, from the picture plane to the station point (Greene, 1983; Sedgwick, 1980). In Figure 2, as in any other one-point perspective, each distance point is the same distance from the LOS as the station point is from the picture plane. 


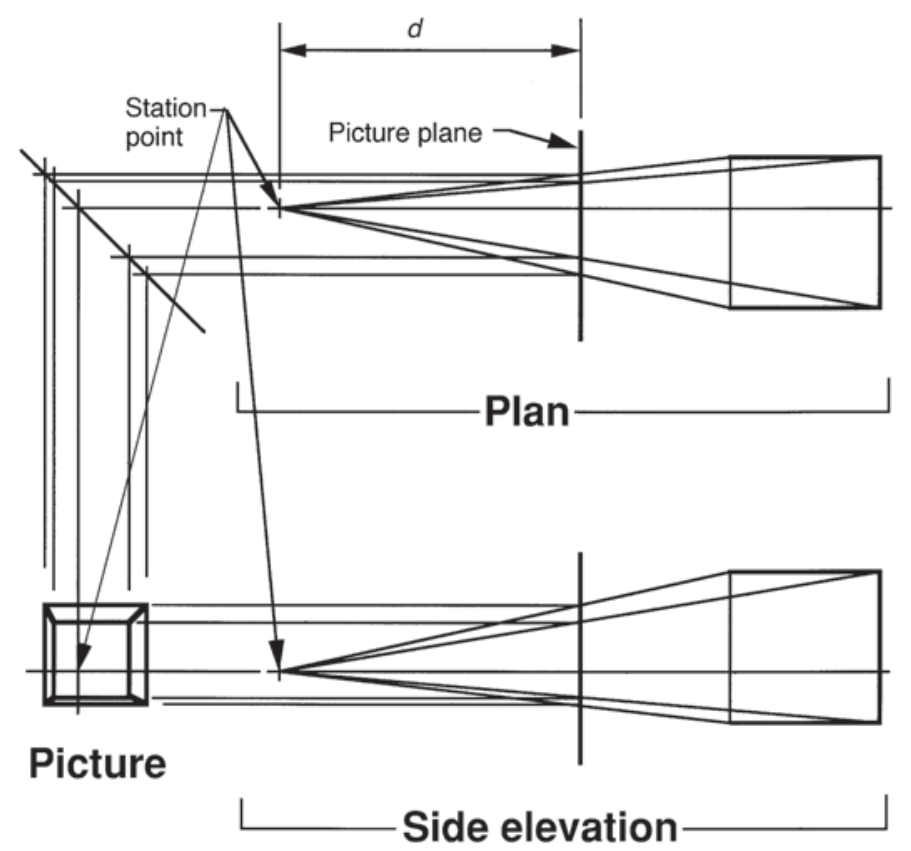

Figure 1. The construction of a picture, in one-point perspective, of a cube-shaped box (that has its open-end forward), from plan and elevation drawings of the box.

\section{Alberti's Veil and the Sightline Method Compared With the Grid}

Unfortunately for finding the generality of station points, many pictures show nothing we could satisfactorily interpret as a ground-plane grid. However, Alberti (1435/1991, pp. 65-67) also suggested another method of drawing (Alberti's veil or Alberti's window), in which a perspective is made by direct projection, analogously to the sightline approach for finding the station point. The simplest version of the veil is a pane of glass placed in the picture plane, on which we trace the outlines of what we see through the glass. As Alberti points out, if we do this, we will realize that the picture that results is specific to its own station point. It follows that the visual array from a wrong station point, projected through the same picture, will indicate a wrong virtual space. Because this is so, we can find the station point with the sightline approach.

The geometric basis of the sightline approach is this: Axiomatically, when a known relation exists in space between an amount in depth and an amount in lateral extent, the depiction of that relation will specify the distance $(d)$ along the LOS, from the picture to its station point. This is true because, at a wrong value of $d$, virtual-space depths are shortened or elongated, relative to virtual-space lateral extents, which remain unchanged. (By definition, depth and laterality extend, respectively, parallel with and perpendicular to the LOS.)

Both the sightline approach and the ground-plane-grid approach depend on the same fundamental properties of perspective. But in the sightline approach, the relational

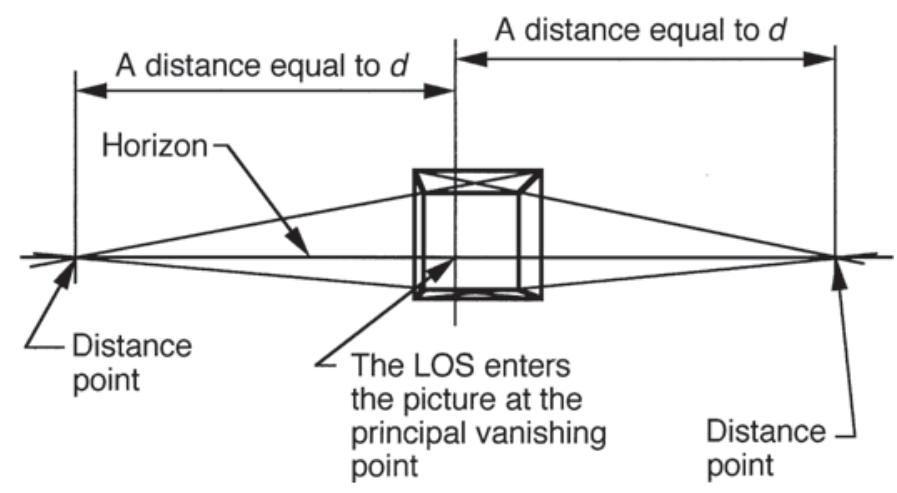

Figure 2. The traditional ground-plane-grid solution for $\boldsymbol{d}$ for the picture of a cube-shaped box that was constructed in Figure 1. 
principle stated above is applied explicitly and more directly, resulting in two considerable advantages over the ground-plane-grid method: The features that are in depthto-lateral relation may be discontinuous or may meet at any angle, and their relation may express linear or angular properties. ${ }^{2}$ In short, the sightline procedures can be more flexibly applied. However, their advantages can sometimes be offset by the need to identify the place where the LOS intersects the picture plane, before we can apply them. (This is not a problem with the ground-plane-grid method, in which the LOS intersection is determined integrally with finding $d$.) The LOS intersects the center of any uncropped photograph, unless perspective-shifting camera equipment was used. And in a one-point perspective, a kind frequently encountered, ${ }^{3}$ it intersects the picture's readily identified single principal vanishing point (Greene, 1983; also see Figure 2). Nonetheless, there are some pictures in which there is no sure way to locate the LOS from the pictures themselves, in which case the sightline approach will not work. (To locate a station point, there must be information of some kind regarding all three orthogonal directions in space. A depth-to-lateral relation in and of itself does not provide this, but, together with the LOS position, it does.)

\section{The Graphic Procedure}

When the requirements for using the sightline approach are fulfilled, one may choose between graphic and computational versions of it. Figure 3 displays the essentials of this approach; only two of the topmost edges of a box identical to the box in Figure 1 are present. As seen by the observer, these are the top rear and top right edges, which form the right-angle figure shown in the plan and elevation. From these views, a picture showing only those two right-angled edge lines is drafted by projection, in the manner of Figure 1. With a picture made in this way, graphic reconstruction by the sightline approach amounts to a direct reversal of the picture-making projections, with the exception that there will almost always be one or more trial reconstructions of plan and elevation views, which, because $d$ is wrong, contain the criterion depth-to-lateral relation incorrectly and in which, therefore, the criterion object or feature set is wrongly shaped.

In Figure 3, the criterion relation that must appear in virtual space and that we must know independently of the picture is, technically, that the $\operatorname{leg} a_{2}$ of the depicted angle extends in depth the same amount as the leg $a_{1}$ extends laterally. We also must know the entry point of the LOS, and this is shown in the picture.

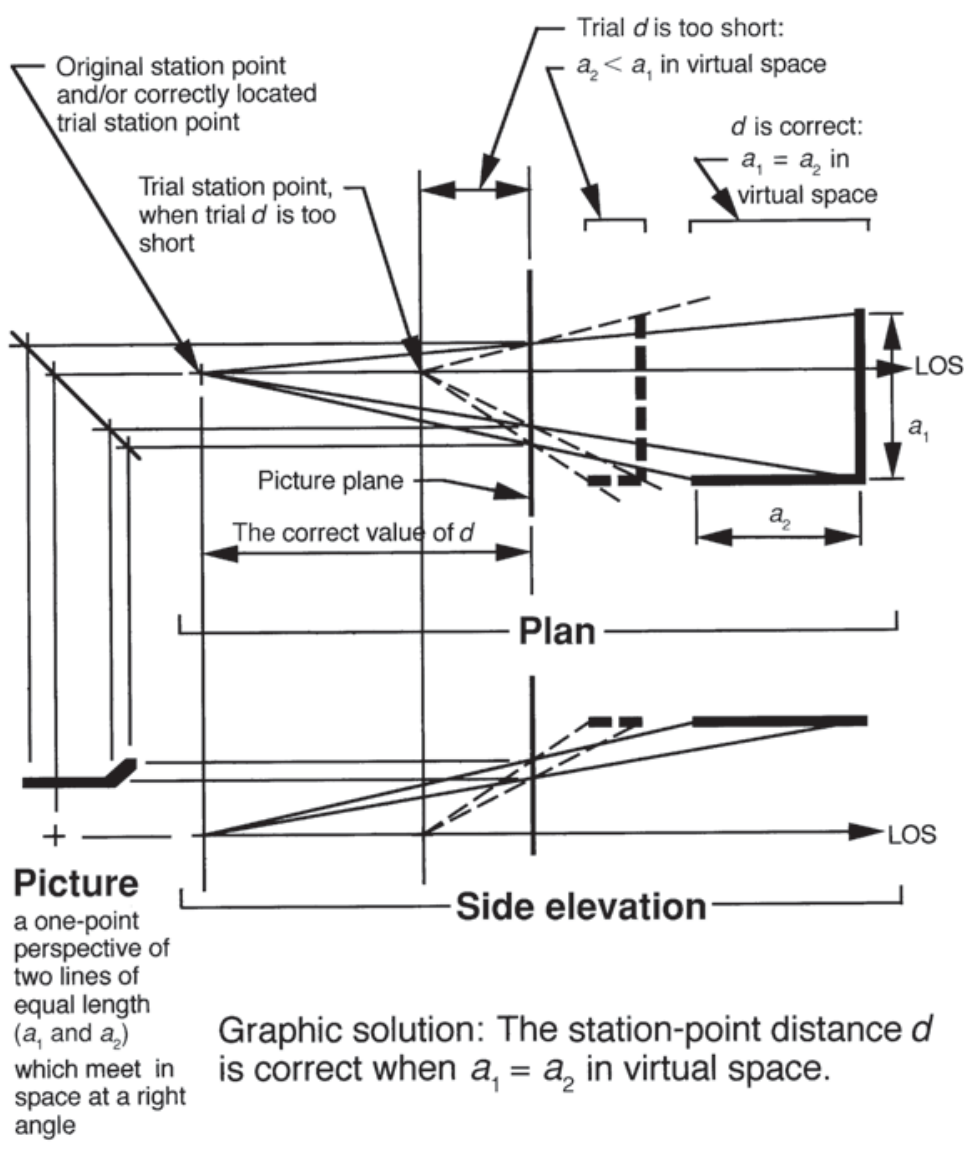

Figure 3. The graphic (projective) sightline procedure for finding $d$ for the picture of a simple right-angled figure (formed from two of the upper edges of the box shown in Figures 1 and 2). 
(We actually know also that this is a right-angle figure, oriented with one leg extending directly forward, and, with this information, we can see that the angle is horizontal in space. Because we know this much, we could have constructed the LOS position from just knowing the level of the horizon, by extending the depiction of $\operatorname{leg} a_{2}$ to meet it. We also could have solved for $d$ using the traditional grid method as in Figure 2; since the picture of the angle is a rudimentary ground-plane-grid element, we could have extended to a distance point the single diagonal the picture defines.)

More programmatically, the steps in a graphicprojection sightline reconstruction of the station point are the following.

1. Identify in the picture a depth-to-lateral relation, the $3-\mathrm{D}$ value of which is known.

2. Using a trial value of $d$, draft a plan-and-elevation orthographic layout, showing edge views of the picture plane with relevant object-contour coordinates marked on them, enough of the virtual space behind the picture to show the criterion depth-to-lateral relation, and the trial station point in front of the picture. (Use a "side" elevation, which presents a direct view of extents along the LOS.) To position in virtual space the object that has or the features that have the critical depth-to-lateral relation, extend visual-array lines from the trial station point through picture-plane coordinates of the object's relevant contour intersections, until the array lines reach positions in virtual space that match the object's known size or distance. If the object's true size/distance is unknown, select either some reasonable size for it or a position for it in space along an appropriate array line. For example, select a size for the lateral leg $\left(a_{1}\right)$ of the angle pictured in Figure 3; position leg $a_{1}$ in virtual space (in plan view) where its array lines have spread apart enough to produce exactly the specified distance $a_{1}$ laterally between them. Draw in leg $a_{2}$ where it will match its own array lines and will abut $a_{1}$ perpendicularly as it should. (Such a layout made without knowing any true object size or distance will yield a correct $d$ but will not determine the scale of the virtual space it reconstructs, since the value of $d$ is independent of the size assumed for the scene. However, $d$ is proportional to the size of the picture itself - that is, to its degree of enlargement or minification from the original.)

3. See whether the layout contains the correct value of the criterion relation. In Figure 3, this means measuring whether $a_{1}=a_{2}$, in the plan view. In general, in reconstructing any pictorial situation, the plan and elevation views must agree completely with respect to the criterion relation and must, taken together, express that relation correctly for $d$ to be considered correct.

4. Assuming the first trial produces an incorrect value for the criterion depth-to-lateral relation, repeat Steps 2 and 3 , using feedback from the first trial as a guide to selecting a more correct trial value of $d$. Often, only one or a very few retrials will be needed. (For maximum accuracy, it sometimes is best to construct a series of layouts that brackets the values for $d$ at which no depth-to-lateral error is revealed, and $d$ is considered correct.)

Iteration is not always necessary and, strictly speaking, might never in principle be required. When the criterion relation is a simple proportion of depth to breadth, the true value of $d$ may be calculated directly from the amount of criterion error that is produced by the (incorrect) first trial value for $d$, since the amount of distortion in virtual depth will be proportional to the amount of error in $d$. When the criterion relation is more complex, however, such as in Figures 5-8 and Figure 10, iterative drafting may be a much easier and faster way than specifying the correctional calculation that should be made.

\section{The Computational Procedure}

Figure 4 shows the derivation of an equation that may be solved for $d$, given the pictorial situation of Figure 3 . This computational procedure requires a slightly different set of steps.

1. As with the graphic solution, identify in the picture a depth-to-lateral relation that is known.

2. Make a plan-and-side-elevation sketch, which need not be to scale, showing enough of the scene being pictured to include the station point, edge views of the picture plane, and the depth-to-lateral relation of interest.

3. Sketch into place all the trigonometric and geometric properties that relate specific picture-plane coordinates to features in virtual space that seem relevant to the depthto-lateral relation being used and therefore might help in finding $d$. For the most part, these will be the properties of similar triangles and/or the Pythagorean relation of the sides of a right triangle with its hypotenuse. More sophisticated algebraic methods involving matrices may sometimes be appropriate, but we will not discuss these here.

4. Express these picture-to-scene relations as equations, at least some of which will involve $d$. The goal is an equation expressing $d$ in terms of picture-plane-coordinate distances only, to be solved for $d .{ }^{4}$ If the depth-to-lateral relation is validly specified, such an equation should exist. Equations will be specific to a pictorial situation and to the depth-to-lateral relation that is identified therein.

\section{When Only the Sightline Procedures Will Work}

Figure 5 is a one-point-perspective picture showing part of a casement window, with a sash swung open from its frame. Assuming we have no independent information about the proportion of sash width to sash height from which to reconstruct a ground-plane grid, the traditional reconstructive methods are of no help in this case. However, this depiction does contain a definable depth-tolateral relation in virtual space, which makes a sightline solution possible, per Step 1: The sash is angled open. The sash presumably fits its opening, so that both have essentially the same real width. But the sash, being opened, has a lateral component to its width in virtual space, unlike its opening, which extends only in depth. Thus, the sash and its opening will be differentially shortened or elongated 


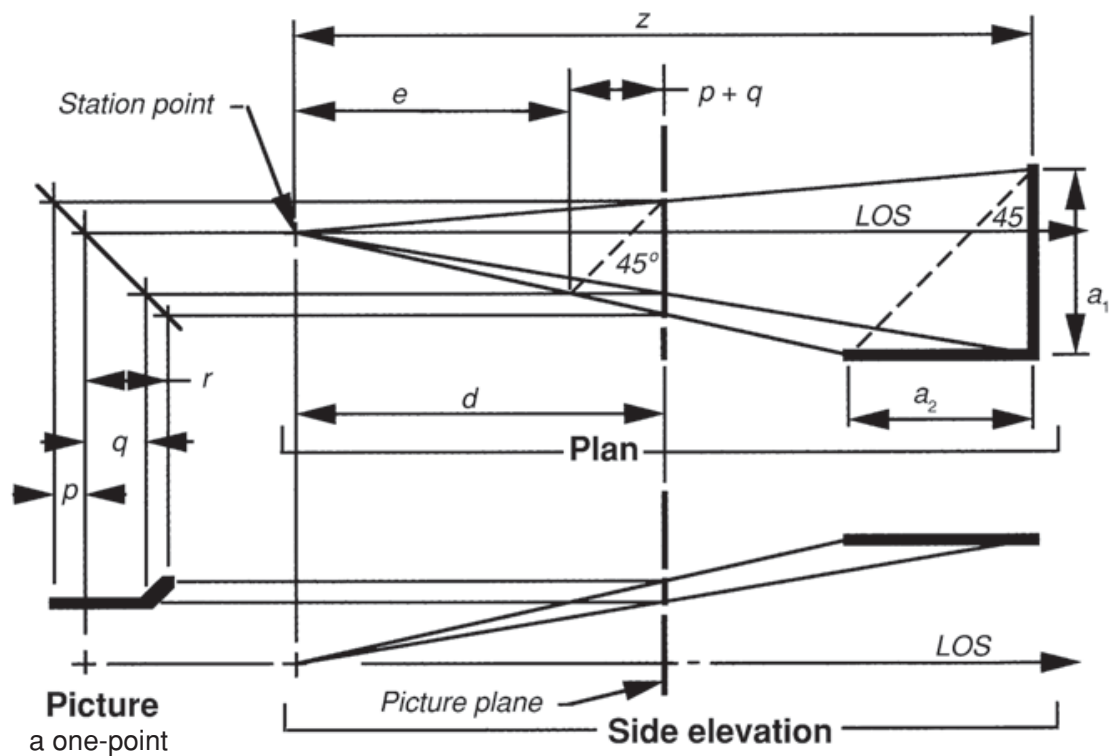

perspective of two lines of equal length $\left(a_{1}\right.$ and $\left.a_{2}\right)$ which meet in space at a right angle

Computational solution: When a depth and a laterality have the relation of two sides of a horizontal square drawn in one point perspective,

$$
d=\frac{p+q}{1-q / r} .
$$

Derivation of the above equation for $d$, given the relation that $a_{1}=a_{2}$ in the virtual space constructed from the original station point:

1. $a_{1}=a_{2}=a \quad 2 . d=e+p+q \quad 3 . q / e=r / d ; d=r e / q$

$2 \& 3 . r e / q=e+p+q$,

which, multiplying through by $q$ and rearranging terms, yields

$e=\left(p q+q^{2}\right) /(r-q)$.

Then, from Equation 3, substituting for $e$,

$$
\begin{aligned}
d & =(p r+q r) /(r-q) \\
& =\frac{p+q}{1-q / r} .
\end{aligned}
$$

Figure 4. The computational (algebraic) procedure of the sightline approach, applied to the picture of a right-angled figure that was shown in Figure 3.

in any virtual depth projected from an incorrect value of $d$. (At any given incorrect value of $d$, the opening will be more shrunk or stretched than will the sash.) Either a graphic or a computational version of the sightline approach may be used to find $d$.

Figure 6 shows the final, successful trial graphic layout, produced by following the steps given for a graphic solution. In Step 2, if nothing is independently known of the window's real size or location in depth, the sash and its opening may be placed in virtual space by assuming either a size in height for the sash and its opening or a difference in height between the top of the opening and the LOS. If the latter amount is assumed, array lines are projected from the trial station point in the elevation view through the picture-plane coordinates for the top corners of the sash's opening (i.e., for the top ends of dimensions $c$ and $e$, as these appear in Figure 5), until they intersect the height in virtual space that is chosen for the opening, which intersections locate the opening in depth. Then, extending lines (perpendicular to the LOS) from the elevation to the plan, the window and wall are located laterally by where these extension lines intersect array lines drawn through planview picture-plane coordinates for the opening's vertical (jamb) edges. Since this is a one-point perspective, these jamb edges should appear in virtual space along a line (representing the wall) that is parallel with the LOS.

When a layout contains the correct criterion relation (Step 3) and therefore the correct $d$, the top open corner of the sash itself, as located in the plan view where the array line through its picture-plane coordinate crosses an 


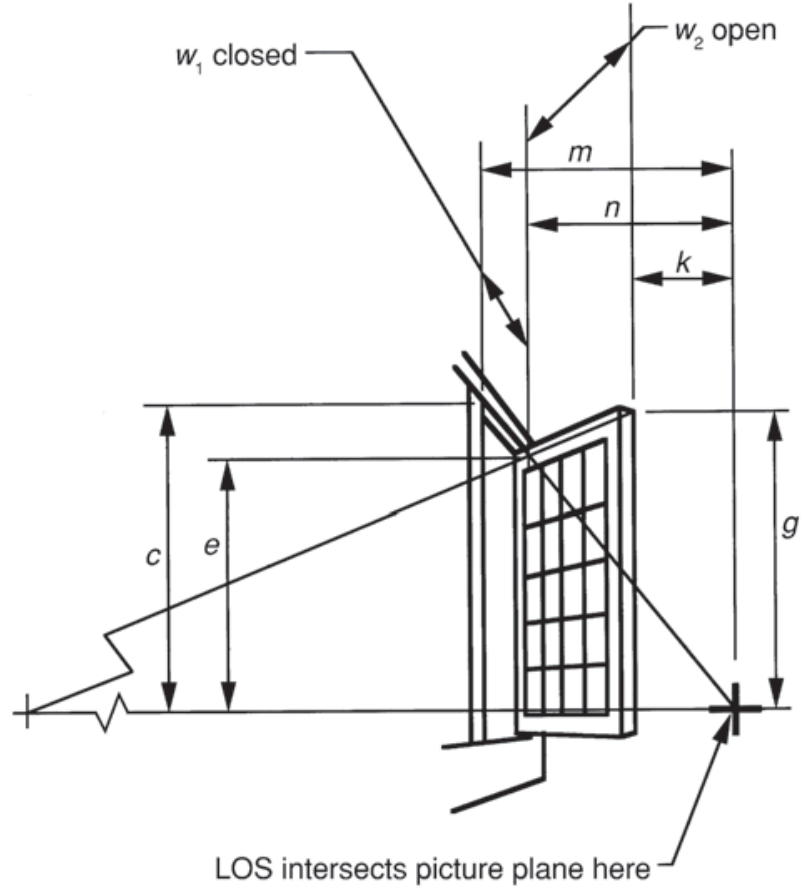

$d$ (not shown) = station-point distance from the picture plane, along the LOS

Figure 5. A picture showing a partially opened casement sash in one-point perspective. (The dimensions are lettered with reference to those in Figure 8.)

arc drawn to represent the corner's trajectory as the sash opens, will be at the same depth in virtual space as when it is located in the elevation view where its array line crosses the top of the sash opening. (A vertical line in Figure 6, drawn from elevation to plan at the depth of the open corner, confirms this.)

Figure 7 shows what happens in virtual space to the width of the sash $\left(w_{2}\right)$ versus the width of its opening $\left(w_{1}\right)$ when $d$ is incorrect: The sash no longer fits its opening. (Figure 7 assumes that the length $w_{2}$ has been established by projection from the elevation view.) Like Figure 5, Figure 7 indicates variable dimensions that refer to Figure 8.

Figure 8 illustrates the computational procedure applied to a not-to-scale plan-and-elevation sketch of the sash and opening in Figure 5. Picture-plane and virtualspace coordinates are related as comparable points in similar triangles formed by array lines, and the sash width $w_{2}$ is the hypotenuse of a right triangle, the other two sides of which are specified by virtual-space dimensions parallel with and perpendicular to the LOS. From these relations are constructed the equations given, from which a resultant equation for $d$ is derived.

Figure 9 is a well-known picture in perspective on which the methods of Figures 5-8 can be used, whereas, as Steadman (2001, p. 80) indicates, traditional methods seem inapplicable.

\section{Two-Point Perspectives: Rectilinearity as a Depth-to-Lateral Relation}

Only in one-point perspectives, where the main object's axes are all either parallel with or normal to the LOS, is the rectilinearity of those axes preserved in virtual spaces reconstructed from the wrong $d$. This means that twopoint and three-point perspectives, unlike the one-point perspective, generally contain a very simple and useful means of finding $d$. Figure 10 illustrates this, with a twopoint-perspective picture of a horizontally positioned cross. When reconstructed using the correct value of $d$, the lines forming the cross meet at $90^{\circ}$ in virtual space; when $d$ is chosen incorrectly, they do not.

The three-point-perspective graphic solution for rectilinearity is more complex than Figure 10, since it requires auxiliary projections. Halloran (1989) shows the sightlinereconstructed geometry of a three-point picture. But such a solution for a three-point perspective may seldom be needed, since there is a nongrid, noniterative traditional way to find $d$ and the LOS for any three-point perspective

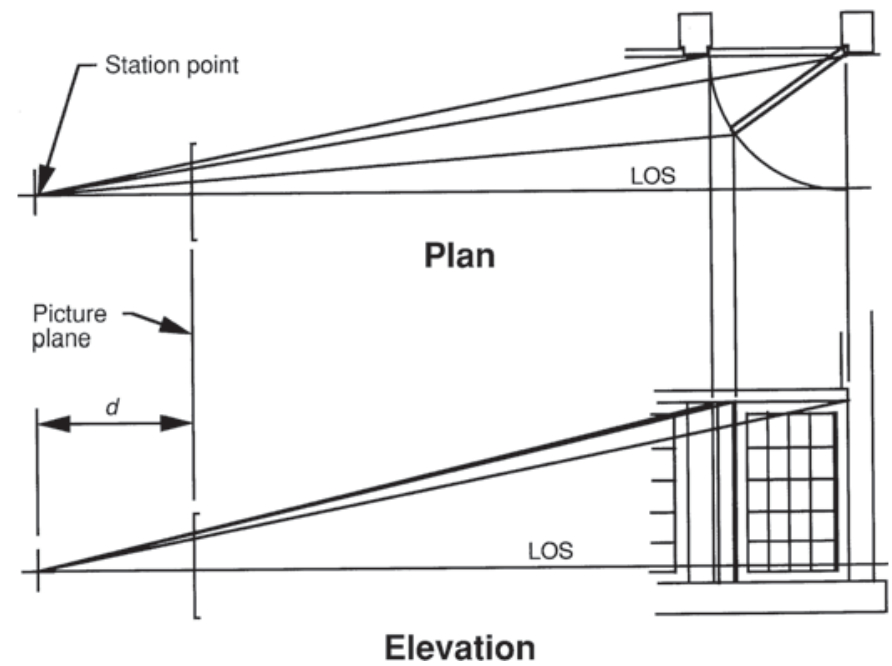

Figure 6. Graphic reconstruction of the window pictured in Figure 5. 


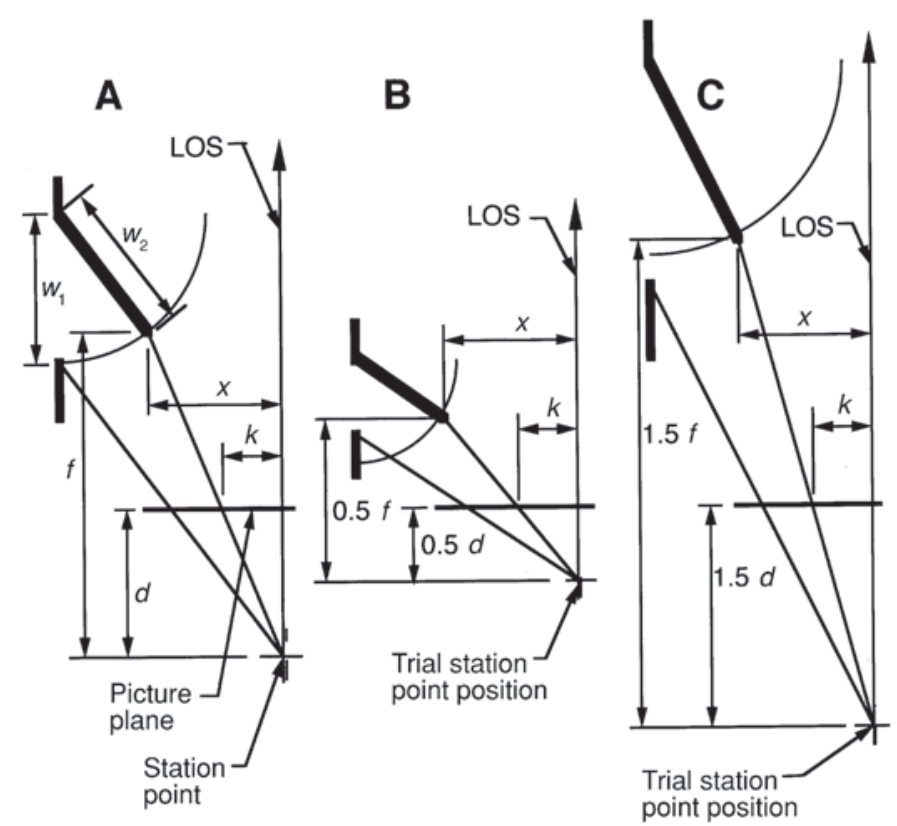

Figure 7. Diagrams of the effect of correct and incorrect values of $d$ on the virtual space reconstructed for the picture of a casement window shown in Figure 5. (A) $d$ is correct; $w_{1}=w_{2}$. (B) $0.5 d$ is too short; $w_{1}<$ $w_{2}$. (C) $1.5 d$ is too long; $w_{1}>w_{2}$.

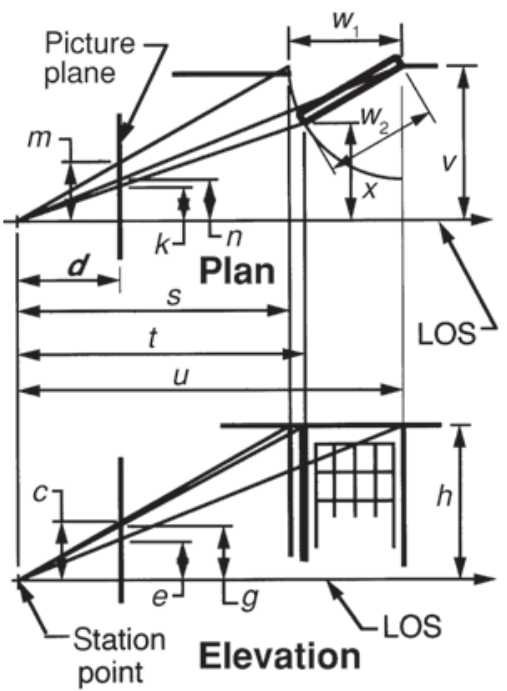

1. $w_{1}=w_{2}=w$

2. $m s=d v$

3. $d v=n u$

4. $k t=d x$

5. $d h=g t$

6. $e u=d h$

7. $g x=h k$

8. $w=u-s$

9. $(u-f)^{2}+(v-x)^{2}=w^{2}$

$$
=(u-s)^{2}
$$

By replacing terms in Equation 9 with terms from Equations 1-8,

$$
\begin{gathered}
\frac{1}{k^{2}}\left(\frac{k d h}{e}-\frac{k d h}{g}\right)^{2}+\frac{1}{d^{2}}\left(\frac{n d h}{e}-\frac{k d h}{g}\right)^{2}=\frac{d^{2} h^{2}(m-n)^{2}}{e^{2} m^{2}} \\
d^{2}(g-e)^{2}+(n g-k e)^{2}=\frac{d^{2} g^{2}(m-n)^{2}}{m^{2}} \\
d=\frac{n g-k e}{\sqrt{\frac{g^{2}(m-n)^{2}}{m^{2}}-(g-e)^{2}}} .
\end{gathered}
$$

Figure 8. Derivation of an equation for the sightline computation of $d$ for any pictorial situation resembling Figure 5. 


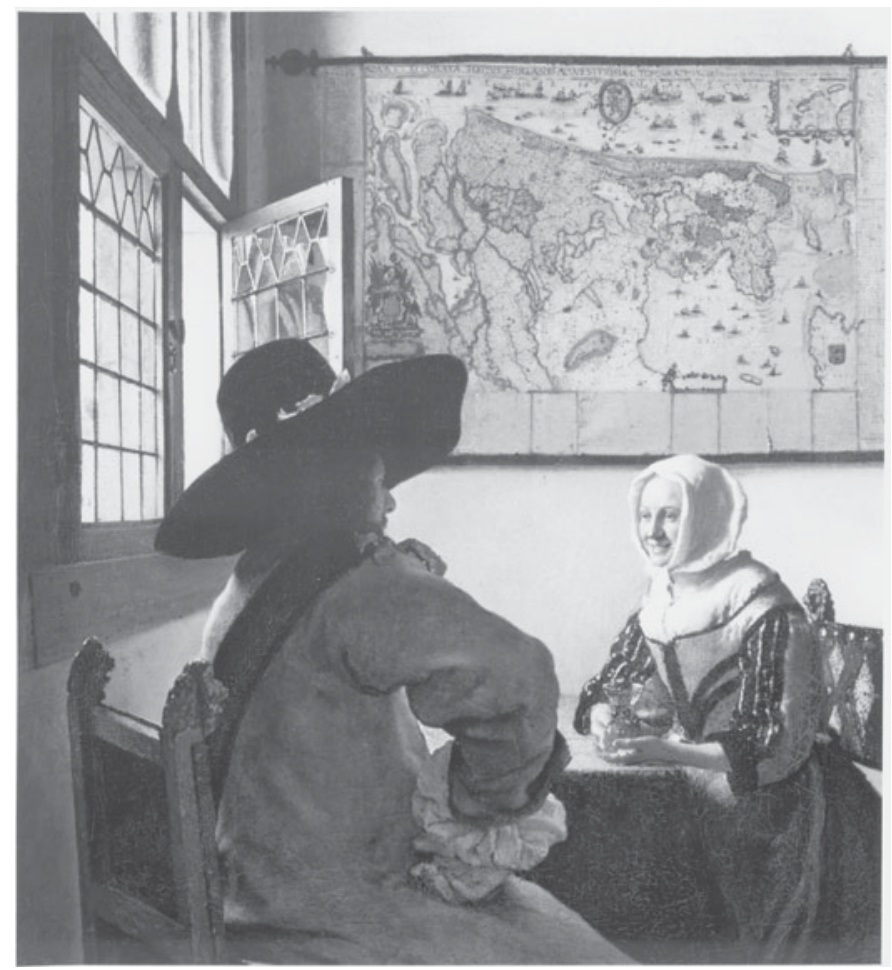

Figure 9. Jan Vermeer, Officer and Laughing Girl. Copyright The Frick Collection, New York.

for which all three main vanishing points are discernible (Greene, 1983).

\section{Differences in the Kind of Result Between Graphic and Computational Procedures}

The choice between graphic and computational sightline procedures may reflect the researcher's skills and inclinations, as well as which procedural solution might seem easier to apply, for a given picture. However, there is also a difference in the nature of the result to be considered. Nominally, the value of $d$ obtained from either procedure would be the same. But graphic layouts tend to produce a small range of values within which $d$ must fall if it is to be considered correct. (This will be the range within which variation in the criterion depth-to-lateral relation is indistinguishable from drafting imprecision.) Computation from an equation, on the other hand, produces a single, discrete value for $d$, but one or more other equivalent equations are possible whenever the relevant perspective information is redundant, and any small errors in measuring pictureplane coordinates might cause other equations to produce slightly different values of $d$. The range of this variability could not be indicated by one equation's result. ${ }^{5}$

\section{Completing the Reconstruction}

The sightline approach is not confined to those pictures for which the entire virtual scene (vs. one depth-to-lateral relation only) can be reconstructed. But once the station point is found, it is usually a straightforward matter to re- construct some or all of the scene. If a ground-plane grid has been employed, the pictured objects may be located directly on the grid as laid out in virtual space (Alberti, 1435/1991, pp. 67-71; Steadman, 2001, p. 77).

Alternatively, and regardless of how the station point was located, array lines constructed from the station point through contour intersections in the picture can be extended into virtual space, in plan and elevation, until they reach the positions where the depicted objects will have their correct sizes, elevations, and distances (Steadman, 2001, p. 78); in other words, the method shown in Figures 3 and 6 for reconstructing the critical depth-to-lateral relation is extended to reconstructing the rest of the scene in virtual space. This is generally what must be done when $d$ has been found by the sightline method (Halloran, 1989, 2004). Unlike when finding $d$, we must know something of the 3-D sizes, shapes, and/or locations of the scene's objects in order to reconstruct their spatial positions.

\section{Some Factors Affecting Accuracy}

When the ground-plane-grid method is used, accuracy depends on the size or extent of the grid as depicted; for the sightline procedures, accuracy depends on the size and particular characteristics of the depth-to-lateral relation. Small errors in measuring the picture can produce disproportionately larger errors in the value of $d$ when the array lines on which the reconstruction depends have a very small included angle with each other or, in the sightline approach, with the LOS. In Figure 6, for example, it would have been 


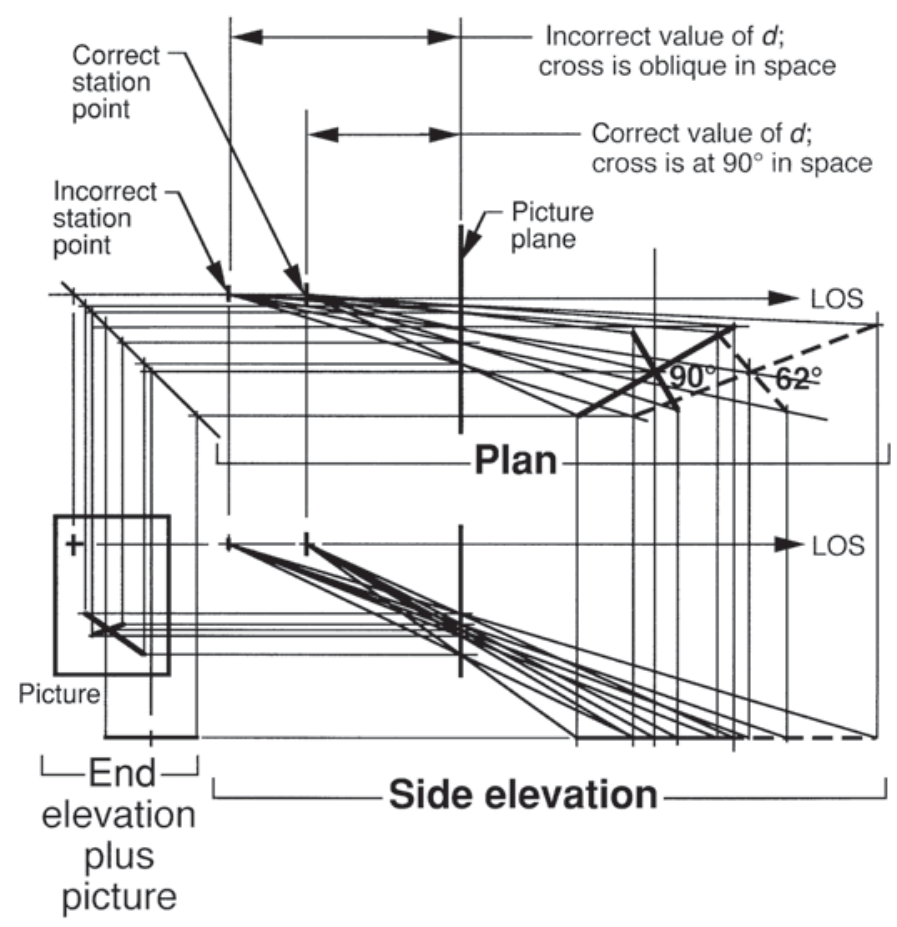

Figure 10. Reconstructions of the virtual space of a picture of a cross drawn in two-point perspective, using a correct value and an incorrect value of $d$.

impossible, as a practical matter, to reconstruct a reasonably accurate value of $d$ using picture-plane coordinates measured for the bottom of the sash shown in Figure 5, rather than the sash-top coordinates that were used, though either choice would, in principle, have been correct.

\section{A Summary Contrast: Sightline Versus Grid in Concept and in Practice}

In the sense employed by Sedgwick (1983), the sightline approach is viewer centered and the ground-planegrid method is environment centered, in that the former fixes directions and distances from the original observer directly, whereas the latter determines positions and orientations in space (grid positions, vanishing points, etc.).

The grid method is often an attractive choice, when it can be used. It assures a noniterative solution, and when an equations is needed, its form is known. The wider applicability of the sightline approach carries with it the burden of finding, in the picture at hand, some usable depth-to-lateral relation, without the configuration of that relation being known beforehand. This need to discover a good depth-to-lateral relation is mostly an issue with onepoint perspectives, since two-point and three-point perspectives contain a relation of rectilinearity, and there is a nongrid traditional solution for the three-point perspective (Greene, 1983), as mentioned.

\section{Broader Implications}

Although originally intended for evaluating pictorial stimuli in vision studies, the sightline approach also has uses in historical, art-historical, and architectural research, where a photograph or perspective rendering of something might be the only dimensional record of it that we have. Concerning vision per se, the discovery that, at least analytically, we are more often able to recover 3-D from 2-D than has been thought may provide insights for machine vision (Beck, Hope, \& Rosenfeld, 1983). And this recoverability of $3-\mathrm{D}$, together with the redundantly overdeterminate nature of perspective stimuli generally (Halloran, 1989, especially p. 473), suggests that static monocular stimuli in general may be more adequate for visual perception than some theories would predict (see Hershenson, 1998, chapters 6-8).

\section{REFERENCES}

Alberti, L. B. (1991). On painting (C. Grayson, Trans.). London: Penguin. (Original work published 1435)

Beck, J., Hope, B., \& Rosenfeld, A. (Eds.) (1983). Human and machine vision. Orlando: Academic Press.

GREENE, R. (1983). Determining the preferred viewpoint in linear perspective. Leonardo, 16, 97-102.

Halloran, T. O. (1989). Picture perception is array-specific: Viewing angle versus apparent orientation. Perception \& Psychophysics, 45, 467-482.

HALlORAN, T. O. (2004). Reconstructing the space in Vermeer's Officer and Laughing Girl. Anistoriton, 8 (Section P043, In Situ, September) Retrieved October 1, 2004, from www.anistor.co.hol.gr/english/index htm.

Hershenson, M. (1998). Visual space perception: A primer. Cambridge, MA: MIT Press.

Pozzo, A. (1989). Perspective in architecture and painting (J. James, Trans.). New York: Dover. (Original work published 1693) 
SEdGWICK, H. A. (1980). The geometry of spatial layout in pictorial representation. In M. A. Hagen (Ed.), The perception of pictures I: Alberti's window: The projective model of pictorial information (pp. 33-90). New York: Academic Press.

SEDGWICK, H. A. (1983). Environment-centered representation of spatial layout: Available visual information from texture and perspective. In J. Beck, B. Hope, \& A. Rosenfeld (Eds.), Human and machine vision (pp. 425-458). Orlando, FL: Academic Press.

StEAdMAn, P. (2001). Vermeer's camera: Uncovering the truth behind the masterpieces. Oxford: Oxford University Press.

\section{NOTES}

1. Perspectives of buildings and other constructed objects are conventionally categorized as one-point, two-point, or three-point, according to how many of the orthogonal axes of the main depicted rectilinear object (or group of aligned objects in a scene) are not perpendicular to the LOS.
2. For an angular relation to be useful to reconstruction requires that at least one side of the included angle be neither parallel with nor perpendicular to the picture plane.

3. Alberti's (1435/1991) and Pozzo's (1693/1989) treatises on perspective, for example, consider only one-point constructions.

4. Ideally, this would be a general equation, applicable to all sufficiently similar pictorial situations, as in Figures 4 and 8. Sometimes, though, it is easier to incorporate the measured numerical picturecoordinate values directly into each equation as it is developed, thereby producing a set of equations that is specific to one picture only, to be solved for $d$.

5. I applied sightline procedures to Figure 5 using values measured on a reproduction of Figure 9. A series of layouts produced a 12-cm range for correct $d$. Values of $d$ from two different equations were $6 \mathrm{~cm}$ apart and well within the layout range.

(Manuscript received May 31, 2004;

revision accepted for publication January 10, 2005.) 